\title{
Experimental Investigation of Cavitation Signatures in an Automotive Torque Converter Using a Microwave Telemetry Technique
}

\author{
C. L. Anderson, L. Zeng, P. O. Sweger, and A. Narain \\ Department of Mechanical Engineering-Engineering Mechanics, Michigan Technological University, \\ Houghton, Michigan, USA
}

J. R. Blough

Keweenaw Research Center, Houghton, Michigan, USA

A unique experimental investigation of cavitation signatures in an automotive torque converter under stall conditions is reported. A quantitative criterion is proposed for predicting early and advanced cavitation in terms of suitable nondimensional pump speeds. The dimensionless pump speed that marks early cavitation is obtained by relating this parameter to the appearance of charge-pressure-dependent pressure fluctuations in the differential pressure transducer readings. The differential pressure transducers were mounted at well-defined locations in the pump passage of a torque converter. The data were transmitted by a wireless telemetry system mounted on the pump housing. Data were received and processed by a ground-based data acquisition system. Automatic transmission fluid exhibited cavitation for charge pressures of 70-130 psi and pump speeds of 1000$2250 \mathrm{rpm}$. Advanced cavitation was marked by operating conditions that exhibited a $2 \%$ or more torque degradation from the converter's noncavitating performance.

For a given family of torque-converter designs and a given transmission fluid, the proposed nondimensional pumpspeed criteria are capable of marking early and advanced stages of cavitation for a range of torque-converter sizes and a range of charge pressures in the torque converter.

Received 25 June 2002; accepted 1 July 2002.

This research was sponsored by GM Powertrain, a division of the General Motors Corporation. The authors express their gratitude to Don Maddock of GM and Glen Barna of IR Telemetrics for technical support.

Address correspondence to C. L. Anderson, Department of Mechanical Engineering-Engineering Mechanics, Michigan Technological University, Houghton, MI 49931, USA. E-mail: cander@mtu.edu
Keywords Cavitation, Microwave telemetry, Torque converters

The automotive torque converter (Maddock, 1991) is a complex turbo machine that consists of three elements: a pump, a turbine, and a stator. It is used in the automatic transmission of a vehicle to transfer power from the engine to the transmission gearing during driving and to effectively unload the engine during idle. It multiplies engine torque during vehicle launch and dampens engine torsional vibration and inertial transients.

Power is transmitted from the engine to the driveline by alterations in the angular momentum of a stream of fluid that constantly circulates between the converter elements. Petroleumbased automatic transmission fluid (ATF) is used as the working medium. A typical automotive torque converter cross-section and flow path are shown in Figure 1. The converter pump is attached to the engine crankshaft and rotates at engine speed.

Fluid flowing between the pump blades is expelled from the pump outlet and immediately enters the turbine. The exiting fluid possesses a high velocity in the direction of pump rotation and consequently a high angular momentum. The turbine blades guide the fluid inward and direct it in the opposite direction of the pump rotation, causing a large change in its angular momentum. This results in a torque on the turbine, which is transmitted to the transmission gearing by the turbine shaft. Upon leaving the turbine, the fluid has a relatively low or even negative (as opposed to pump rotation) tangential velocity and angular momentum. The stator receives this fluid and accelerates it back in the direction of the pump's rotation. Once again, the accompanying change in angular momentum produces torque on the stator, and this torque is resisted by a nonrotating shaft built into the transmission housing. Finally, fluid reenters the pump, carrying with it momentum in the direction of the pump rotation. As a consequence of the flow vectoring by the stator, pump 


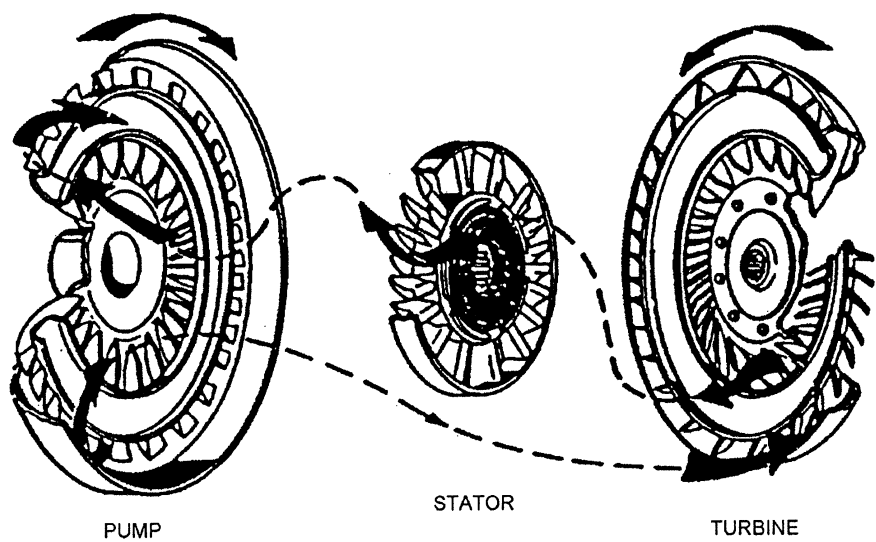

FIGURE 1

Components and working-fluid flow path in a typical automotive torque converter.

torque is less than turbine torque (torque is "multiplied"), and this difference is exactly equal to the stator torque.

In all three elements, pressure forces between the blades and the working fluid accomplish the change in angular momentum. The blades bend the streamlines of the flow, and this action elevates the static pressure on the leading (pressure) side of the blade while dropping the pressure on the trailing (suction) side. The total element torque is the product of the pressure difference across the blade and the radius from the shaft center line, integrated over the blade surfaces. When torque into the converter pump increases, as when the engine throttle is opened, leading and trailing side pressures are forced higher and lower, respectively. At some level of input torque, the pressure on the suction side of the stator blades drops below the vapor pressure of the fluid, allowing the fluid to cavitate.

\section{GENERAL CONSIDERATIONS}

\section{Torque Converter Cavitation}

Cavitation is expected at any location in a flowing liquid where the local pressure is at or below the vapor pressure of the liquid at the local temperature. Local vaporization of the liquid at any nucleation site then causes the development of a vaporfilled bubble, or cavity, in the flow. As the cavities move into regions where pressure is higher than the local vapor pressure, a combination of compression and rapid condensation of the vapor in the cavity causes the collapse of the cavity. At the point of disappearance of the cavity, the pressure momentarily rises to a high value, leading to subsequent pressure fluctuations. The amount of cavitation depends on the mean absolute pressure levels in the flow, and in this investigation a representative value is taken to be the torque converter's charge pressure. This dependence distinguishes cavitation-induced pressure fluctuations in an incompressible flow from those that arise from rotating ma- chinery or turbulence-induced fluctuations which, necessarily, are independent of the mean absolute pressure levels.

Cavitation is suppressed by pressurizing the torque converter torus with ATF from the transmission's hydraulic control system. This pressure, commonly termed converter charge pressure, is superimposed on the pressure field of the turbomachine and consequently raises the torque, at which point cavitation occurs.

Cavitation is to be avoided in automotive torque converters for two reasons. First, noise caused by even mild cavitation is noticeable in the passenger compartment of most vehicles and is of such a nature that it can cause customer complaints. The noise can be audible at light throttle, low charge-pressure conditions, when there is little engine noise to mask it, and during heavy throttle acceleration. Second, the disruption of the converter's normal speed-torque characteristics allows the engine to run in regions of its own speed-torque envelope where it is not expected to operate. This is most often noticed as inconsistent vehicle performance or excessive transmission temperature.

Because the effects of cavitation are important in other fluid machinery too, there is a large amount of literature that deals with the broad subject of cavitation and its effects on walls (e.g., Knapp, 1970; Rheingans, 1962). There are also a large number of fundamental studies that deal with bubble or bubble population dynamics in cavitation (e.g., Ceccio and Brennen, 1992; Kumar and Brennen, 1993; Plesset and Prosperetti, 1977). This article's study of cavitation signatures in automotive torque-converters is, however, more in the spirit of identifying cavitation in pumps through suitable cavitation numbers and net positive suction head (NPSH) values. While we are not aware of cavitation studies of a torque converter, some relevant cavitation studies of pumps are Ardizzon and Pavesi (1994); Franz and colleagues (1990); and Hashimoto and colleagues (1997).

\section{Detection of Cavitation}

Laboratory evaluation of cavitation in automotive torque converters has generally been limited to the determination of the pump torque at which the converter deviates from the ideal parabolic pump speed versus the pump torque curve. This curve is usually obtained at stall condition and at constant charge pressure and temperature. The results have not been completely satisfying because the large net power input to the converter forces rapid heating. This creates pressure, torque, and speed transients that exceed the typical test equipment's capabilities to detect cavitation-induced variations. Noise evaluation has been even less successful because background noise in the test cell overwhelms any noise generated by the converter.

The approach taken in this work is to associate pressure fluctuations in the working fluid of the converter with the onset and development of cavitation. If the point of a cavity collapse is close to a boundary wall, the resulting pressure spikes can first be recorded by a pressure transducer before an effect on the mean values of the flow variables (torque) can be detected.

A zone in the flow field that includes the formation, maintenance, and disappearance locations of the cavities is called a 
cavitation zone. Converters operating under low torques and high charge pressures will not cavitate and would have no cavitation zones. Conversely, high-input torques and low charge pressures produce large, strong cavitation zones (associated with the size and number of the cavities). These conditions produce a measurable departure from the noncavitating speed-torque characteristic. Between these two conditions there is a range in which cavitation zones are formed but do not have sufficient influence on the flow to produce an identifiable deterioration of the speedtorque characteristic. For the purposes of this study, conditions that induce a $2 \%$ loss of pump torque at a given pump speed are termed advanced cavitation. Conditions that exhibit abnormal pressure fluctuations but do not support a $2 \%$ torque degradation are termed early cavitation.

\section{Telemetry}

The pressure measurements were acquired from the rotating torque converter using a wireless telemetry technique (Zeng, 2000). A $2.5-\mathrm{GHz}$ carrier wave was used to transmit the transducer information. The technique typically uses conventional transducers and a microwave transmitter on the moving part. The evolution of the signal is shown in Figure 2.

A voltage-to-frequency conversion was performed on the output of the transducer. The $0-100 \mathrm{kHz}$ voltage-to-frequency converter is very stable and can be operated across a wide range of temperatures. The resulting signal was then used to modulate a voltage-controlled oscillator $(2.5 \mathrm{GHz})$. The transducer information was thus superimposed on the carrier wave and transmitted

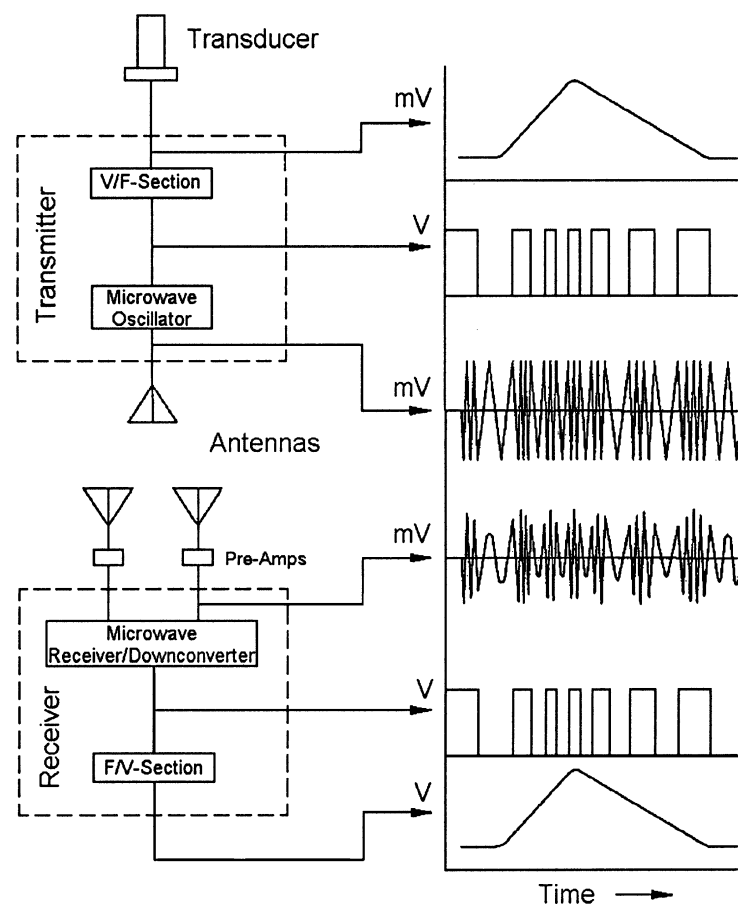

FIGURE 2

Evolution of the microwave telemetry signal.

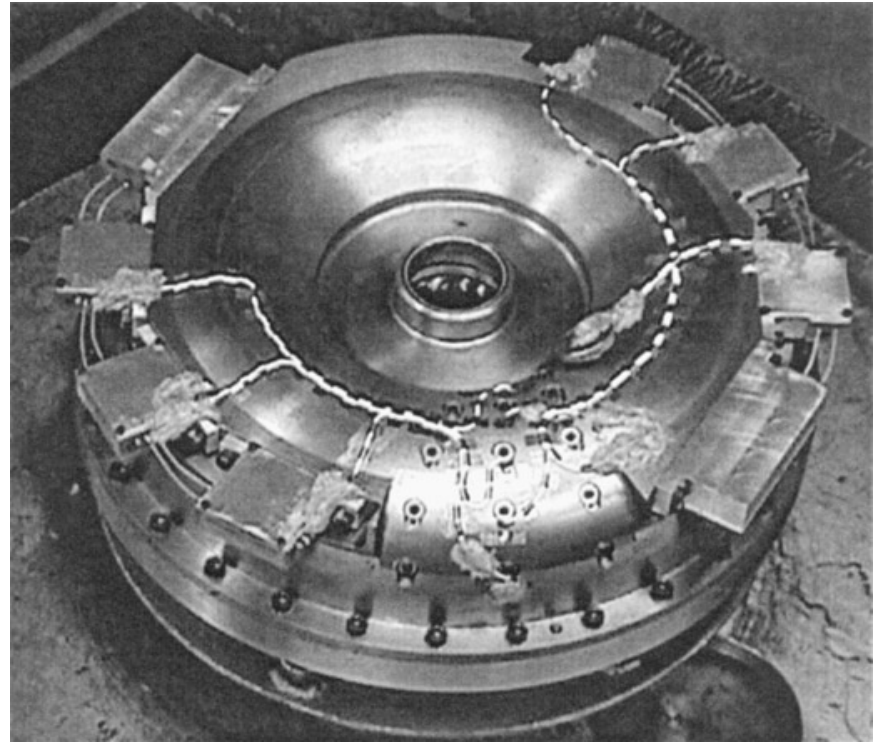

FIGURE 3

Telemetry packages mounted on the torque converter pump.

from the moving part using a dipole antenna milled into the transmitter's housing.

The signal was received by a pair of stationary antennas, down-converted, and processed by an external receiver to reconstruct the original frequency modulation. An analog frequencyto-voltage conversion was performed at the output of the receiver to reconstruct the transducer signal. This final frequencyto-voltage conversion typically requires a low-pass filter with a cut-off frequency of three kHz.

The wavelength of the transmitted signal is small enough that it can easily propagate through the open spaces inside rotating and reciprocating machinery such as engines and transmissions. The 2.5-GHz carrier wave transmitted from the moving part fills either the bell housing or, in the case of this investigation, the laboratory test fixture with the signal. The microwave transmitter and the battery packs that power the electronics are shown mounted on the pump in Figure 3.

\section{CAVITATION SIGNATURES}

At a specified transducer location, a transducer's pressure response, $p(t)$ is composed of a steady pressure component, $\bar{p}$, and a fluctuating pressure component, $p^{\prime}(t)$, as

$$
p(t)=\bar{p}+p^{\prime}(t) .
$$

We expected to find cavitation signatures in the fluctuating pressure signal, $p^{\prime}$. The fluctuating pressure $p^{\prime}$ was obtained with the help of a differential pressure transducer whose reference tube was routed back to the transducer's sensing location, as shown in Figure 4. The diameter and the length of the reference tube was chosen to ensure that the fluctuating component $p^{\prime}$ acting at the reference tube opening was significantly damped by 


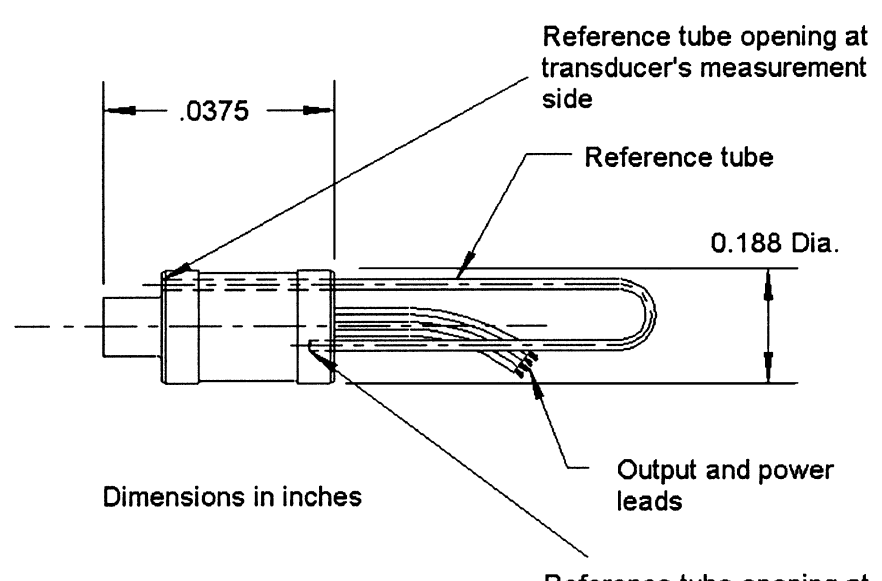

Reference tube opening at transducer's reference side

FIGURE 4

Differential pressure transducer used in torque converter experiment to measure $p^{\prime}$.

the time this signal reached the reference side of the diaphragm. This fact was experimentally established (Zeng, 2000). In this configuration $\bar{p}+p^{\prime}$ acted on the measurement side of the transducer's diaphragm and $\bar{p}$ on its reference side. Thus the voltage signal obtained from the $10 \mathrm{psi}$ differential pressure transducers truly represented $p^{\prime}$.

In principle one might expect to characterize cavitation signatures (the strength of the fluctuating pressure) using a mean square pressure (MSP), where

$$
\operatorname{MSP}=\left(\int_{o}^{T} p^{\prime 2} d t\right) / T
$$

is a representative square amplitude nearly independent of the duration, $T$, of the fluctuating pressure, $p^{\prime}$. However, for these experiments, it was preferable to use a frequency-filtered MSP that retains only a frequency range $f_{1} \leq f \leq f_{M}$ of the signal $p^{\prime}$ that is relevant to cavitation. To define frequency-filtered MSP, first we converted the time domain signal $p^{\prime}$ over $0 \leq t \leq T$ to the frequency domain by obtaining a discretized version of the Fourier Transform $P(f)$ :

$$
P(f)=\frac{1}{T} \int_{0}^{T} p^{\prime}(t) e^{-i 2 \pi f t} d t
$$

The $P(f)$ values were replaced by $P_{i}(f)$ values, which are the Fast Fourier Transform of pressure signals $p_{i}^{\prime}$ for $1 \leq i \leq N$ obtained by dividing the original $p^{\prime}$ signal of duration $T$ (here, $T=2.3 \mathrm{sec}$ ) into $N$ equal records (here, $N=20$ ) of duration $T / N$. Then, in order to get a clearer spectrum by reducing random noise, an ensemble-averaged power (EAP) in the frequency domain was defined as

$$
\operatorname{EAP}(f)=\frac{\left(\sum_{i=i}^{N}\left|P_{i}(f)\right|^{2}\right)}{N} .
$$

We wanted the filtered MSP to be a measure of signal energy over a resolvable frequency range, $f_{1} \leq f \leq f_{M}$, such that its value was independent of record duration $T$ and the number $N$ of subrecords obtained from the original signal record of duration $T$. For these reasons, power summation (PS) was defined as

$$
\mathrm{PS}=(T / N) \int_{f_{1}}^{f_{M}} \operatorname{EAP}(f) d f \cong \sum_{i=1}^{M} \operatorname{EAP}\left(f_{i}\right)
$$

where $M$ is the number of frequency data points of EAP for a given scan rate, $S$ (here, $S$ is 20,000 samples per sec), duration $T$, and number of divisions $N$ of the original record length $T$. Typically, the highest frequency $\left(f_{M}\right)$ is less than $f_{\text {Nyquist }}=S / 2$ and $f_{1}>N / T$. A restriction on $f_{M}$ was imposed by a lowpass filter imbedded in the receiver of the telemetry system depicted in Figure 2. Both theoretically and computationally, it was established that the PS defined in Equation (5) is reasonably independent of scan rate, record length $T$, and number of divisions $N$ of the original record length $T$. It should be noted that both the MSPs and PS have units of $\mathrm{Pa}^{2}$ and units of $\mathrm{psi}^{2}$.

For a stall condition (fixed turbine) and a steadily rotating pump (at rotation rate $\Omega$ ), any time-averaged local flow variable $\Phi$ (such as MSP, PS, etc.) at a point inside a torque converter would depend on quantities that typically determine the solution of the torque converter's boundary-value problem. Therefore, for cavitating flows, one must add charge pressure, $p_{c}$ (the pressure at the inlet of the torque converter), and vapor pressure, $p_{v}$ (at a representative flow temperature), to the list of parameters that typically determine the flow field in noncavitating flows. It is easy to see that the variable $\Phi$ depends on the shape and size (size is determined by pump diameter, $D$ ) of the torque converter; the location of the point in the flow field where $\Phi$ is measured; pump speed, $\Omega$; charge pressure, $p_{c}$; vapor pressure, $p_{v}$; representative mean values of fluid density, $\rho$, and viscosity, $\mu$, at a representative temperature; and pressure difference, $\left(p_{c}-p_{e}\right)$, or through-put oil flow rate, $Q$, between the inlet and the exit of the torque converter. As an approximation, it can be assumed that the pressure difference, $p_{c}-p_{e}$, is determined primarily by the pump speed $\Omega$ and only secondarily by the through-put flow rate, $Q$. It can be further assumed that, at reasonably fast pump speeds, the Reynolds number $\left(\rho D^{2} \Omega\right) / \mu$ is large, so the effects of viscosity, $\mu$, are negligible (as is the case for centrifugal pumps under fully rough turbulent-flow conditions). Therefore, for a given family of torque converters, a given fluid (ATF), and a given transducer location, the more significant variables affecting $\Phi$ are given by the assumption

$$
\Phi \cong \operatorname{function}\left(D, \Omega, \rho, p_{c}\right) .
$$


The dimensionless form of Equation (6) for $\Phi=\sqrt{\mathrm{PS}}$ is easily obtained (see П-Theorem in Munson et al., 1998) and is given by

$$
C a^{\prime} \cong \operatorname{function}\left(\tilde{\Omega}_{p}\right)
$$

where $C a^{\prime}$ is a nondimensional fluctuating pressure, which we refer to as the fluctuating cavitation number and is defined as

$$
C a^{\prime}=(\sqrt{\mathrm{PS}}) /\left(\frac{1}{2} \rho(D \Omega)^{2}\right)
$$

and $\tilde{\Omega}_{p}$ is the dimensionless pump speed, defined as

$$
\tilde{\Omega}_{p} \equiv(D \tilde{\Omega}) /\left(\sqrt{p_{c} / \rho}\right) .
$$

It should be recognized that at small values of pump speeds $\Omega$, experimentally obtained $C a^{\prime}$ values may not exactly follow Equation (7) as the Reynolds number $\rho D^{2} \Omega / \mu$ or an equivalent viscosity parameter such as $D \sqrt{\rho p_{c} / \mu}$ should be included in addition to $\tilde{\Omega}_{P}$ in the argument list of Equation (7).

\section{EXPERIMENTAL PROCEDURES}

\section{Test Apparatus}

The torque converter tests were performed in a laboratory fixture. The fixture housed the converter and supported both the input and the output shafts. A separate hydraulic unit supplied ATF to the fixture and provided control over the flow rate, temperature, and charge pressure. The inlet pressure (charge pressure), inlet temperature, outlet temperature, and outlet pressure of the converter were measured.

All of the data reported in this investigation were acquired in the stall condition. This condition simulates the initial stages of vehicle launch, a condition in which cavitiation is sometimes observed. During stall conditions, the toroidal flow through the blade elements is the greatest, and the separation region on the suction side of the stator blade is expected to be at its lowest pressure.

The transducers were installed behind static pressure taps in the pump. It was expected that cavities would form on the suction side of the stator blade and move with the toroidal flow into the pump. The cavities were expected to collapse somewhere in the pump passage as the pressure of the ATF increased. Static pressure taps were located at five different stations as shown in Figure 5. The taps were located along the passage's center line. The pressure taps are shown schematically in Figure 5 to be located in the same pump passage. However, in the actual test converter, the pressure taps were located in adjacent passages.

\section{Test Matrix and Data Acquisition}

In this study, pump speed and charge pressure were varied over ranges that were believed to adequately cover conditions that might be encountered in actual operation. Pump speed was

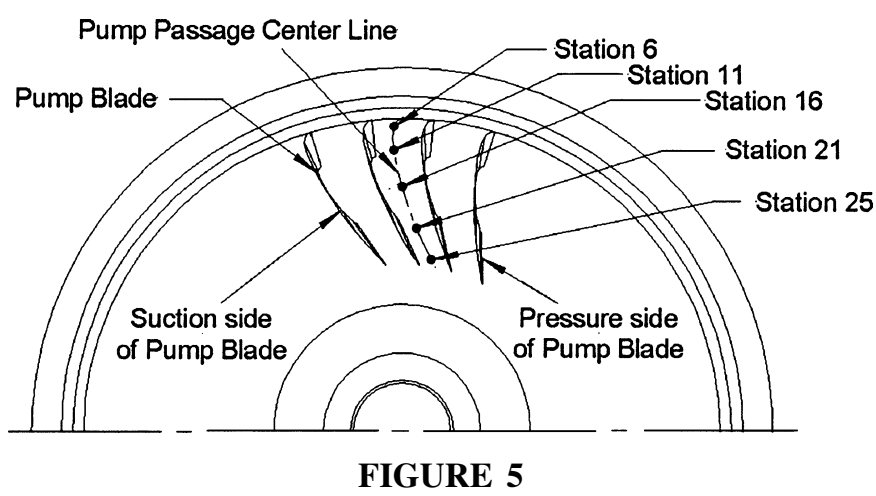

Radial locations of static pressure taps.

varied from 500 to $3000 \mathrm{rpm}$ in increments of $250 \mathrm{rpm}$. Charge pressure was varied from 70 to $130 \mathrm{psi}$ in increments of $10 \mathrm{psi}$. At all test conditions the inlet temperature was held constant at $80^{\circ} \mathrm{C}$, and the outlet temperature would settle to an equilibrium temperature based on the amount of power input to the oil. Data were acquired at steady-state conditions as long as the outlet temperature was below $120^{\circ} \mathrm{C}$. At higher pump speeds, data acquisition was initiated as soon as the outlet temperature exceeded $120^{\circ} \mathrm{C}$ in order to protect the electronics and batteries.

Five single-channel transmitters were mounted on the pump for the pressure measurements. The data were transmitted simultaneously at slightly different carrier frequencies. Each transmitter was paired with its own receiver, which down-converted the signal and reconstructed the transducer output. Data were acquired using a PC-based data acquisition system.

\section{RESULTS AND DISCUSSION}

Various $p^{\prime}(t)$ signals at the specified pressure transducer locations were obtained by converting the transducer's raw real-time voltage signal through proper calibrations (Zeng, 2000). Representative $p^{\prime}$ signals from the transducer at station 25 for charge pressure $\left(p_{c}\right)$ values of 130 and $70 \mathrm{psi}$, respectively, at a pump speed of $1750 \mathrm{rpm}$, are shown in Figure 6.

As stated earlier, the fluctuating pressure $\left(p^{\prime}\right)$ in these two figures could have three causes: (1) flow fluctuations arising from mechanical forcing functions associated with shaft rotation, various blade passages, etc.; (2) flow turbulence; and (3) the collapse of vapor cavities typically associated with cavitation. Of these three phenomena only cavitation is strongly influenced by charge pressure. Figure 6 clearly indicates the increasing trend of the fluctuating pressure when the charge pressure was reduced from $130 \mathrm{psi}$ to $70 \mathrm{psi}$. This is indicative of cavitation. Such cavitation signatures would now be quantified and related to the flow variables through proper processing of the fluctuating pressure $\left(p^{\prime}\right)$ data.

For the signals in Figure 6, we obtained $|P(f)|$ defined in Equation (3) and $\operatorname{EAP}(f)$ defined in Equation (4). These are shown in Figures 7 and 8, respectively. The $\operatorname{EAP}(f)$ values for each of the charge pressures tested are included in Figure 8. An increase in $\operatorname{EAP}(f)$ with decreasing charge pressure can be 

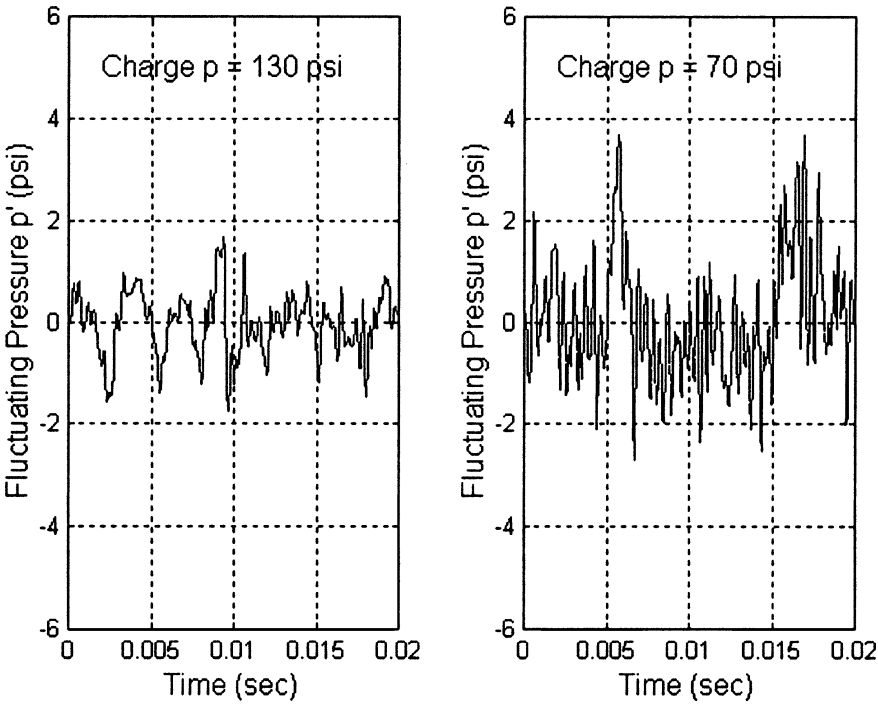

FIGURE 6

A representative pressure signal, $p^{\prime}$, at station 25 and a pump speed of $1750 \mathrm{rpm}$.

observed in Figure 8. This trend is what one would expect to see in a cavitation signature as charge pressure decreases. The signature had a fairly broad spectral distribution. The roll-off in $\operatorname{EAP}(f)$ above $3000 \mathrm{~Hz}$ was caused by the effects of the low-pass filter in the microwave receiver.

For each charge pressure and pump speed tested, the $\operatorname{EAP}(f)$ values were then summed from $f_{1} \sim 0 \mathrm{~Hz}$ to $f_{M}=4000 \mathrm{~Hz}$ to obtain PS through its definition in Equation (5). The resulting values of PS as a function of pump speed and charge pressure are shown in Figure 9. The charge-pressure dependency began to be clear above line MB. We refered to this as early cavitation because, as is shown later, there was no noticeable effect on the pump's performance when it was measured at the dynamome-
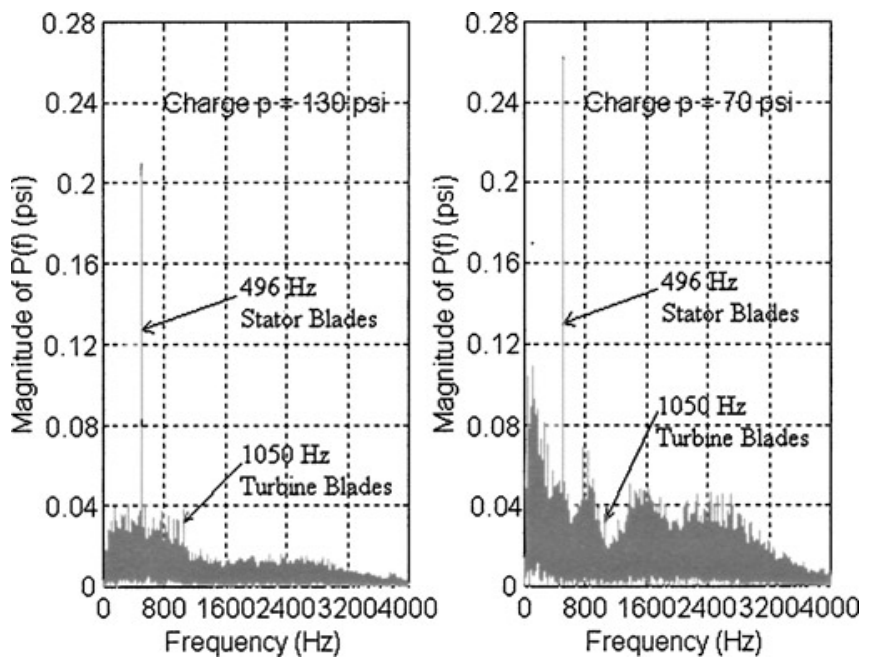

FIGURE 7

$|p(f)|$ values of data in Figure 6.

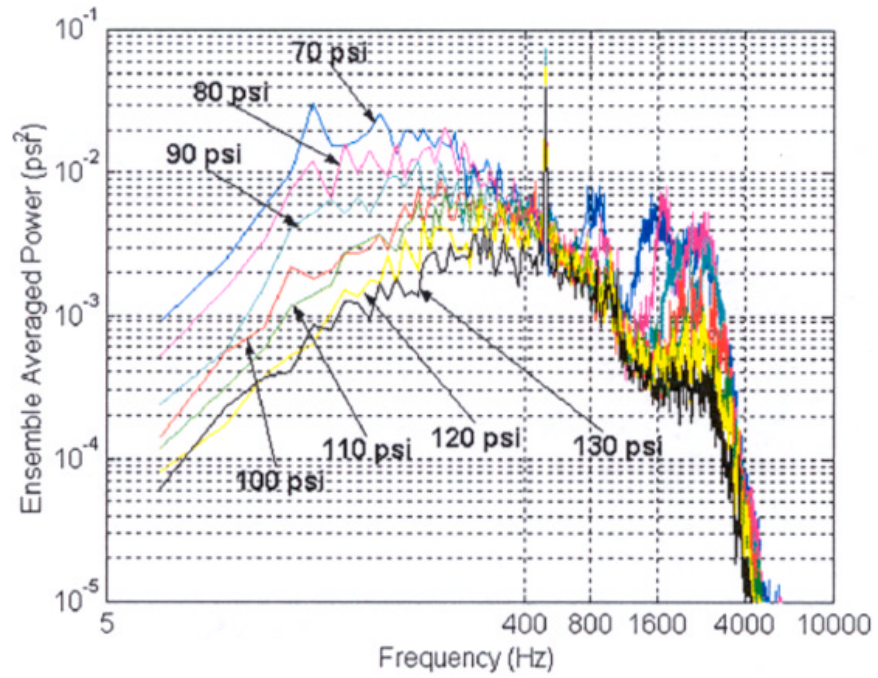

FIGURE 8

$\operatorname{EAP}(f)$ values for $N=20$; various charge pressures at $1750 \mathrm{rpm}$.

ter. Yet from the in situ measurements, we saw a noticeable onset of charge-pressure dependency in the region above line MB.

Next, PS values obtained for Figure 9 were used to obtain the fluctuating cavitation number $\mathrm{Ca}^{\prime}$ in Equation (8) and the results were plotted, with the help of the nondimensional pump speed $\tilde{\Omega}_{p}$, in the form suggested by Equation (7). This resulted in a relationship of the type shown in Figure 10.

Clearly, in Figure 10, there is only an approximate single curve (which appears to be linear) of the type suggested by Equation (7) in the range $1.32<\tilde{\Omega}_{p}<1.76$. All the data in this region did not collapse perfectly into a single curve because of experimental errors and perhaps because we neglected the nondimensional parameter that represents $p_{c}-p_{e}$

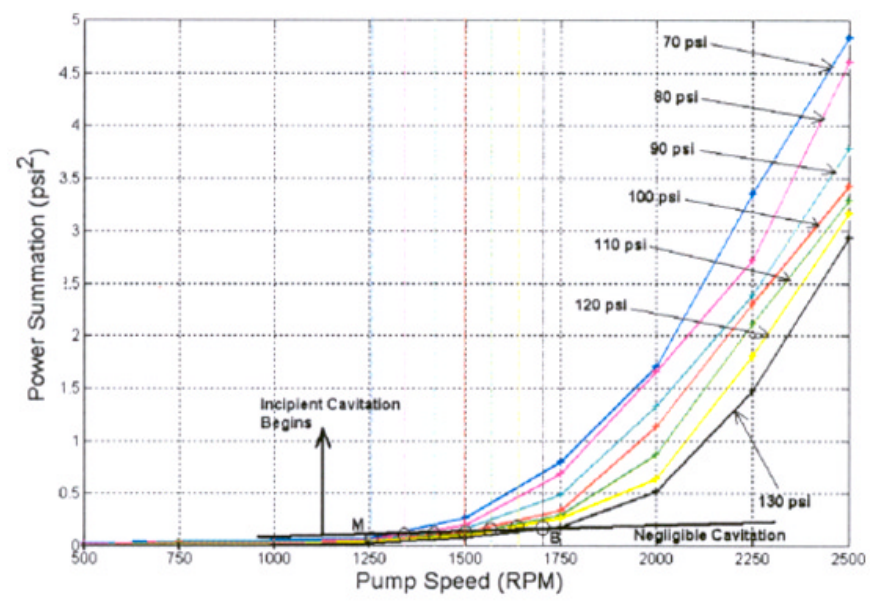

FIGURE 9

PS values as a function of pump speed and charge pressure. 


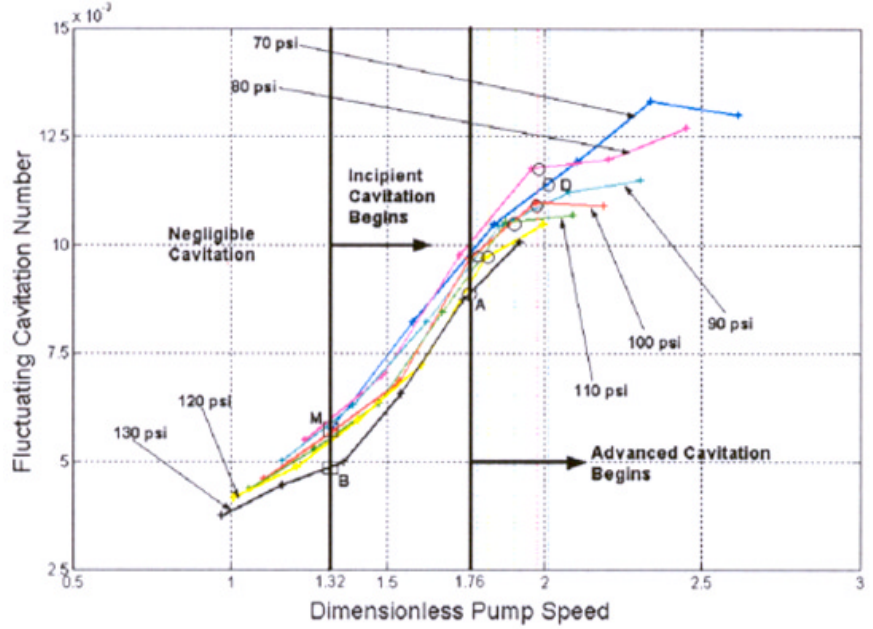

FIGURE 10

Fluctuating cavitation number, $C a^{\prime}$, as a function of dimensionless pump speed at station 25 .

to account for variations in throughput flows. At lower pump speeds, the effects of nondimensional viscosity and, at higher pump speeds, the effects of fluid property variations with time and temperature were expected to enhance departure from the primary relationship indicated by Equation (7). Despite this, considering the enormous complexity of the flows, Figure 10 justifies the use of Equation (7) for the sought-after cavitation signatures.

The charge-pressure dependency began approximately above line MB in Figure 9. When these points were transformed and superposed on power summation curves in Figure 10, line MB of Figure 9 transformed into a vertical line $\mathrm{MB}\left(\tilde{\Omega}_{p} \approx 1.32\right)$, and the above-MB zone of Figure 9 mapped to $\tilde{\Omega}_{p}>1.32$. This implies that the proposed early cavitation criteria of $\tilde{\Omega}_{p} \approx 1.32$ is reasonable.

To define an advanced cavitation regime, we looked at the pump's torque-versus-pump-speed performance with the help of stall-condition $K$-factor values, where $K \equiv \Omega / \sqrt{T_{p}}$. For noncavitating stall situations, the $K$ factor is known to be a constant (Maddock, 1991). The noncavitating value for the torque converter used in the test facility is 220.5 and is shown on the torque curve in Figure 11. A 2\% degradation in torque, also shown in Figure 11, can routinely be distinguished in dynamometer testing and for that reason was chosen as the criterion for advanced cavitation.

To mark this situation, for each charge pressure, we plotted $K$-factor values against nondimensional pump speed $\tilde{\Omega}_{p}$ (Fig. 12). In Figure 12, line AD corresponds to $2 \%$ torque degradation. Thus, depending on charge-pressure values, advanced cavitation occurred over a range of nondimensional pump speeds $1.76<\tilde{\Omega}_{p}<2.01$. Therefore, a conservative criterion for advanced cavitation, which is valid for the entire test matrix, is recommended to be $\tilde{\Omega}_{p}>1.76$.

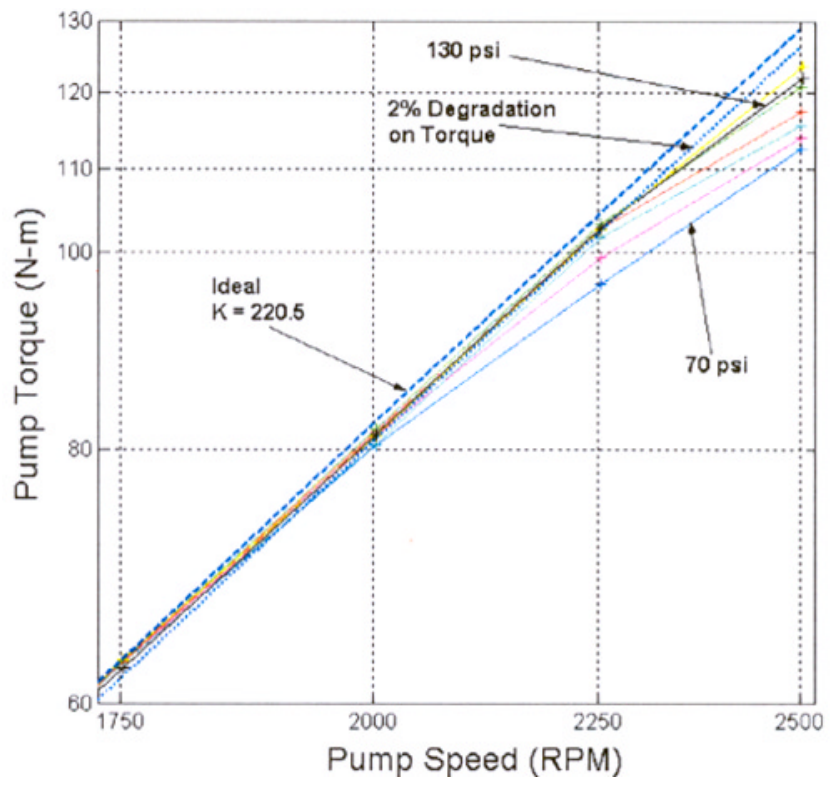

FIGURE 11

Pump torque as measured at the input shaft for various charge pressures.

In our experiments, we also found that the peak strength of the cavity's collapse zone was located closer to the pump inlet because the value of PS decreased for transducers located at increasing radial distances from the center of the pump (Zeng, 2000, Fig. 4.23).

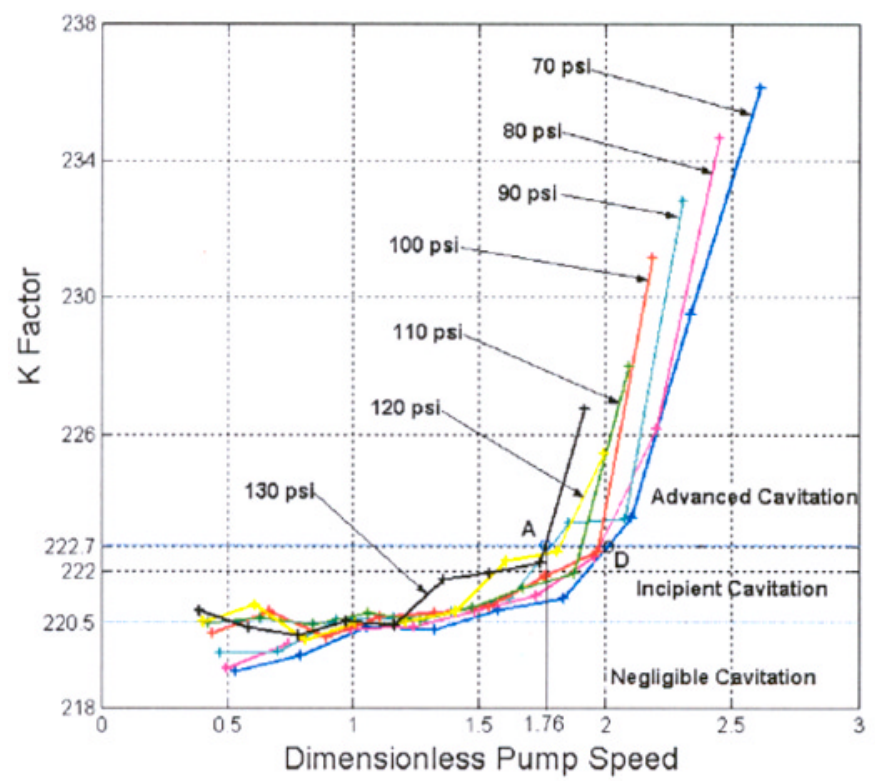

FIGURE 12

$K$ factor as a function of dimensionless pump speed at station 25 . 


\section{CONCLUSIONS}

Using microwave telemetry and the identification of pressure fluctuation signals, it is possible to infer the inception of cavitation in a torque converter before standard dynamometer testing.

Analysis in the frequency domain can separate, if necessary, the effects of blade passage frequencies and allows various restrictions on the threshold values of the frequency.

The power summation in the frequency domain provides a useful measure of cavitation signatures.

Nondimensional pump speed can be directly and successfully used in practice to identify early cavitation for a range of charge pressures.

\section{NOMENCLATURE}

$\mathrm{Ca}^{\prime} \quad$ Fluctuating cavitation number

$D \quad$ Diameter of pump

$f \quad$ Frequency

PS Power summation

$K \quad K$ factor

MSP Mean square pressure

$p^{\prime} \quad$ Fluctuating pressure

$p_{c} \quad$ Charge pressure

$p_{e} \quad$ Exit pressure

$\rho \quad$ Density of automatic transmission fluid

$\Omega \quad$ Pump speed, rad/sec

$\tilde{\Omega}_{p} \quad$ Nondimensional pump speed

$\mu \quad$ Viscosity

$T_{p} \quad$ Pump torque

$\Phi \quad$ Local flow variable

\section{REFERENCES}

Ardizzon, G., and Pavesi, G. 1994, Summer. Numerical prediction and experimental evaluation of cavitation inception on centrifugal pump impellers. Proceedings of the ASME Fluids Engineering Division: Cavitation and Gas-Liquid Flow in Fluid Machinery and Devices, 190:71-77.

Ceccio, S. L., and Brennen, C. E. 1992. Dynamics of attached cavities on bodies of revolution. Journal of Fluids Engineering 114:93-99.

Franz, R., Acosta, A. J., Brennen, C. E., and Caughey, T. K. 1990. The rotordynamic forces on a centrifugal pump impeller in the presence of cavitation. Journal of Fluids Engineering 112:264-271.

Hashimoto, T., Yoshida, M., Watanabe, M., Kamijo, K., and Tsujimoto, Y. 1997. Experimental study on rotating cavitation of rocket propellant pump inducers. Journal of Propulsion and Power 13:488-494.

Knapp, R. T., Daily, J. W., and Hammitt, F. G. 1970. Cavitation. New York: McGraw-Hill.

Kumar, S., and Brennen, C. E. 1993. Some nonlinear interactive effects in bubbly clouds. Journal of Fluid Mechanics 225:565-591.

Maddock, D. 1991. Application and Design of Automotive Torque Converters. Ypsilanti, MI: GM Powertrain, General Motors.

Munson, B. R., Young, D. F., and Okiishi, T. H. 1998. Fundamentals of Fluid Mechanics, 3rd ed. New York: John Wiley.

Plesset, M. S., and Prosperetti, A. 1977. Bubble dynamics and cavitation. Annual Review of Fluid Mechanics 9:145-185.

Rheingans, W. J. 1962, Proceedings of Symposium on Erosion and Cavitation. Philadelphia: American Society for Testing and Materials.

Tillner, W., Fritsch, H., Kruft, R., Lehmann, W., Louis, H., and Masendorf, G. 1993. The Avoidance of Cavitation Damage. London: Mechanical Engineering Publications.

Zeng, L. 2000. Experimental Investigation of Cavitation Signatures in an Automotive Torque Converter. (MSME thesis, Michigan Technological University). 

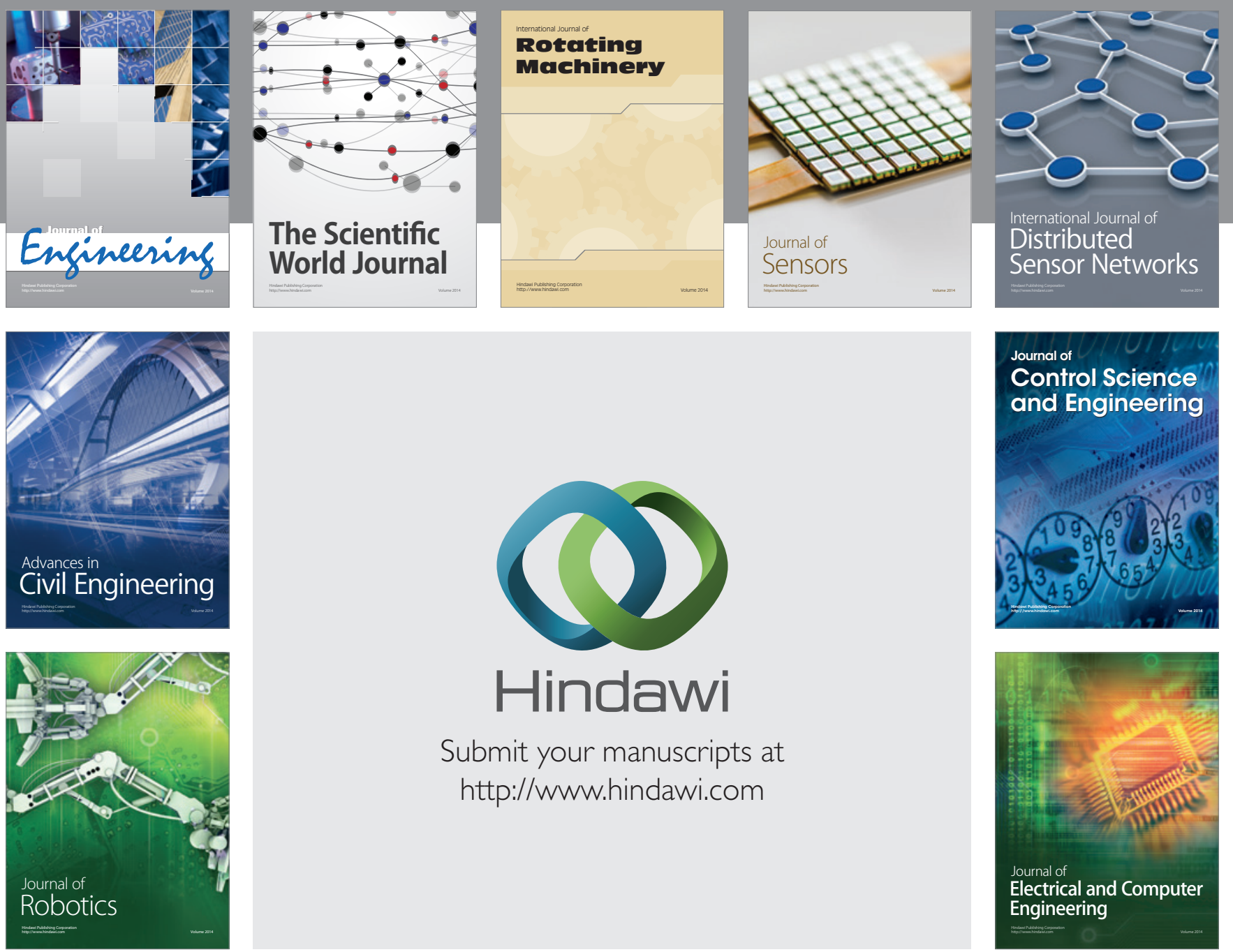

Submit your manuscripts at

http://www.hindawi.com
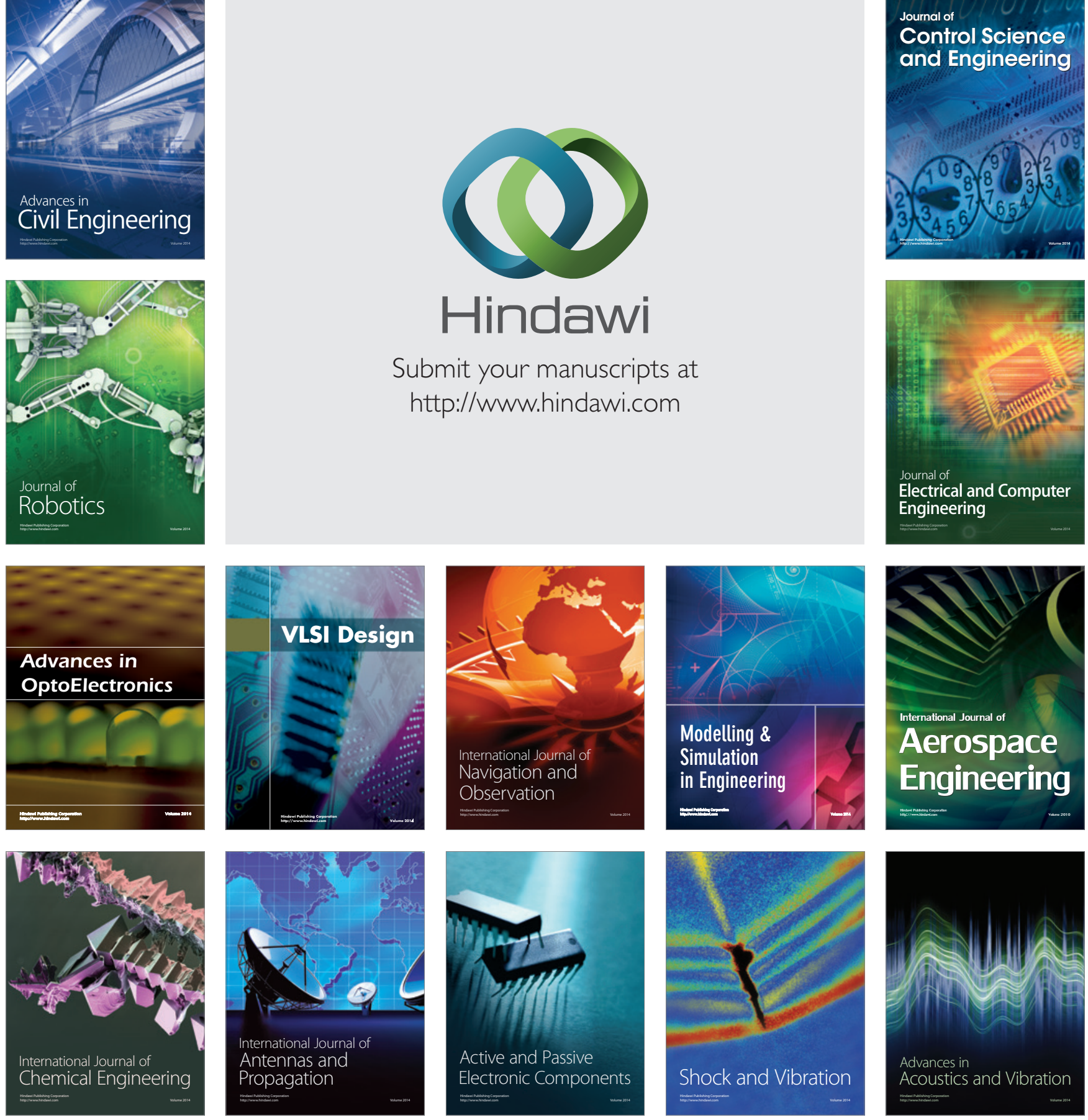